\title{
Moving Horizon Estimation and Control for an Industrial Gas Phase Polymerization Reactor
}

\author{
John D. Hedengren and Kenneth V. Allsford \\ Advanced Process Control \& Optimization \\ PAS, Inc. \\ 16055 Space Center Blvd. \\ Houston, TX 77062 \\ USA \\ Jasmeer Ramlal \\ Sasol Polymers \\ Division of Sasol Chemicals Industries, Ltd \\ 56 Grosvenor Road, Bryanston \\ Randburg 2125 \\ South Africa
}

\begin{abstract}
Moving horizon estimation (MHE) has been applied to an industrial gas phase polymerization reactor to improve estimates of current states and parameters. MHE is compared to Implicit Dynamic Feedback $\left(\text { IDF }^{\mathrm{TM}}\right)^{1}$. With MHE, there is improved estimation of unmodeled disturbances in the UNIPOL ${ }^{\mathrm{TM}}$ polyethylene plant. The UNIPOL ${ }^{\mathrm{TM}}$ technology is licensed by Univation, a joint venture between ExxonMobil and Dow. The polymerization reactor and plant model is a largescale set of differential and algebraic equations (DAEs) posed in open equation form. The DAE model is converted to algebraic equations by orthogonal collocation and solved with the MHE objective function in a simultaneous optimization. NOVA ${ }^{\mathrm{TM}}$, an active-set sparse NLP solver, is used to converge the problem that has 46,870 variables, 18 complementarity conditions, and a Jacobian sparsity of $0.01 \%$. This large, sparse optimization problem is initiated every 5 minutes to update the model as new plant measurements become available and prior to the control optimization. The same plant model is used for nonlinear model predictive control (MPC) with 10 manipulated variables (MVs) and 26 controlled variables (CVs). In this case, a significant advantage is that with MHE a simpler rigorous model suffices for the application of nonlinear MPC.
\end{abstract}

\section{INTRODUCTION}

Model-based calculations and measurements of the process they represent may differ because of model inaccuracies. The reconciliation of model and measurements is an important precursor to be able to use the model for predictive analysis. Predictive analysis is used for fault detection, product quality assurance, model-based control, and to remain within safety or environmental constraints. These model-based techniques require an accurate estimate of the current system states to take advantage of the predictive

\footnotetext{
To who correspondence should be addressed: E-mail:john.hedengren@exxonmobil.com

Authors John D. Hedengren and Kenneth V. Allsford are currently associated with ExxonMobil and Honeywell, respectively.

${ }^{1}$ Unless otherwise indicated, all trademarks listed in this paper are of PAS, Inc.
}

capabilities of the model. Without accurate state estimation, the predictive advantages of model-based calculations cannot be fully utilized. Many approaches have been suggested to accomplish the reconciliation of model and measurements, with a range of trade-offs [1]. Generally, the trade-offs are centered on model form and size, computational expense, ease of implementation, robustness, and off-set free estimation [2] [3].

Unmodeled features of a physical process pose a limitation, in particular, for model-based control. Before optimized manipulated variable (MV) moves can be computed, the current model states must reasonably match the measurements from the actual process. Qin and Badgwell [4] surveyed the current state of nonlinear model predictive control (MPC) applications. According to the survey, the most popular feedback techniques at the time were constant and integrating output bias disturbances. However, Froisy concluded that state estimation is a key enabler that allows linear MPC to reject disturbances more effectively than a well tuned PID controller [5]. Two alternatives of state estimation are the Kalman filter and moving horizon estimation (MHE).

A number of evaluations have compared the Kalman filter to MHE [6] [7] to show that MHE consistently outperforms the extended Kalman filter in the presence of constraints. State estimation of real systems may include changing measurement frequencies and missing measurements, multiple measurements at different sampling frequencies, measurement delay, large-scale nonlinear models, and constraints. MHE, as a part of nonlinear dynamic data reconciliation [8] [9], is flexible to deal with these complications [10]. MHE is a computationally tractable approximation to the optimal infinite horizon estimation [11]. All of the challenges of real system state estimation are naturally handled in the MHE framework. An estimate of the current states is typically obtained by solving a least squares optimization 
problem subject to the model constraints and inequality constraints that represent bounds on variables or equations. Liebman et al. was the first to propose a simultaneous solution approach for MHE where the differential equations are transformed into algebraic equations through orthogonal collocation on finite elements [8]. Later, Albuquerque and Biegler improved the solution approach by exploiting the MHE SQP structure to achieve linear computational scaling with horizon length [12]. Further computation improvement was proposed by Hedengren et al. in an explicit solution to a simplified nonlinear MHE problem [13].

Researchers from ExxonMobil Chemical Company have published MHE results using the NOVA ${ }^{\mathrm{TM}}$ solver for diluent inventory tracking [9] and a liquid phase polymerization reactor [14]. These studies have shown the success of MHE as applied to models of industrial processes.

Many of the previously referenced papers on MHE discuss novel theoretical aspects but do not address some practical issues for industrial applications. The contribution of this work is to discuss another industrial application of MHE including practical issues in implementation, introduce $\mathrm{IDF}^{\mathrm{TM}}$ for the first time, and discuss how $\mathrm{IDF}^{\mathrm{TM}}$ and MHE can be used to improve nonlinear MPC.

\section{Feedback Strategies}

In this paper, MHE is compared with Implicit Dynamic Feedback (IDF $\left.{ }^{\mathrm{TM}}\right)$. Both of these feedback technologies are available as options in the Polymer NLC ${ }^{\mathrm{TM}}$ model predictive control package. IDF ${ }^{\mathrm{TM}}$ originated with DOT Products and was subsequently used by PAS to incorporate model feedback of measured states.

\section{A. Implicit Dynamic Feedback (IDF $\left.{ }^{T M}\right)$}

The $\mathrm{IDF}^{\mathrm{TM}}$ formulation is a feedback technology which estimates disturbances that may be linearly or nonlinearly related to the predictions of the measured state variables. $\mathrm{IDF}^{\mathrm{TM}}$ is a pairing of these measured states with unmeasured disturbance variables. The disturbance variable is adjusted to eliminate plant/model mismatch. The $\mathrm{IDF}^{\mathrm{TM}}$ formulation is compact enough as to be incorporated simultaneously with the control problem within a single history interval.

$\mathrm{IDF}^{\mathrm{TM}}$ is a proportional integral (PI) controller that is integrated with the process model. The PI controller input is the error between the measured state $\left(y_{s}\right)$ and model state $\left(y_{m}\right)$. The output is an unmeasured disturbance variable $(d)$ of the model. This disturbance variable is adjusted proportional to the current and integrated measurement error.

$$
d=K\left(y_{s}-y_{m}\right)+\frac{K}{\tau_{I}} \int_{t=0}^{T}\left(y_{s}-y_{m}\right) d t
$$

The tuning parameters for $\mathrm{IDF}^{\mathrm{TM}}$ are $K$ and $\tau_{I}$, the same as a PI controller. Using a large value of $\tau_{I}$ and small $K$ has the affect of heavily filtering the error term for feedback. In this case the algorithm will take longer to match the plant. Using these tuning parameters and knowing the quality and types of measurements enables trading off of "speed of tracking the plant" versus "stability concerns". A unique feature of the $\mathrm{IDF}^{\mathrm{TM}}$ approach is the method of simultaneous disturbance estimation and prediction, because the model calculated value is computed simultaneously with the disturbance variable calculation. The current model-predicted value of the measured state variable is computed by integration from the last time step from the previous value of the measured state variable. This means the $\mathrm{IDF}^{\mathrm{TM}}$ calculations are automatically decoupled. If the speed at which state variable disturbances are updated is not limited by the frequency of the measurements, the decoupled $\mathrm{IDF}^{\mathrm{TM}}$ approach may be tuned more aggressively than traditional feedback methods that use output disturbances.

$\mathrm{IDF}^{\mathrm{TM}}$ has been successfully used for six years to provide online estimation measurement biases, catalyst activities, kinetic parameter adjustment factors and heat transfer coefficients. However, the IDF limitations are:

1) Past horizon length of one

2) Requirement of equal number of disturbance variables and measurements

3) Inability to handle disturbance variable constraints

\section{B. Moving Horizon Estimation}

The MHE objective function is posed as a minimization of L1 and L2-norm errors to reconcile the model with measured values. Similar to $\operatorname{IDF}^{\mathrm{TM}}$, the disturbance variables $(d)$ are the only degrees of freedom to adjust model predictions to match measurements.

$$
\begin{aligned}
& \min _{d} \Phi=\left\|\frac{y_{s}-y_{m}}{y_{m}}\right\|_{Q_{y}}^{2}+\left\|\frac{y_{s}-y_{m}}{y_{m}}\right\|_{\nu}+\|d-\hat{d}\|_{Q_{d}}^{2} \\
& \text { s.t. } \quad 0=f(\dot{x}, x, u, d) \\
& \quad y_{s}=g(x, u, d) \\
& \quad a \geq h(x, d) \geq b
\end{aligned}
$$

where subscript $s$ refers to sample values, and subscript $m$ refers to model values, $\Phi$ is the objective function value, $y_{s}$ is a vector of measurements at all nodes in the orthogonal collocation structure $\left(y_{s, 0}, \ldots, y_{s, n}\right)^{T}, y_{m}$ is a vector of model values at the sampling times $\left(y_{m, 0}, \ldots, y_{m, n}\right)^{T}, Q_{y}$ is optimally the inverse of the measurement error covariance, $\nu$ is a vector of weights for the absolute errors, $f$ is a vector of model equation residuals, $x$ represents the model states, $u$ is the vector of model inputs, $d$ is the vector of model parameters or unmeasured disturbances, $\hat{d}$ is the vector of previous unmeasured disturbances, $Q_{d}$ is a matrix for the weight on changes of disturbance variables, $g$ is an output function, $h$ is an inequality constraint function, and $a$ and $b$ are lower and upper limits, respectively.

Note that the full MHE problem would allow violation of the state constraints and estimation of the initial states. In such a formulation, state continuity constraints are relaxed and violations are penalized in the objective function. The optimization problem found in Equation 2 does not allow state transition error because the state equations are exactly satisfied at a converged solution [15]. State estimation is accomplished by adjusting past values of disturbance variables. This approach greatly reduces the number of optimization 
degrees of freedom while converging to a solution that is consistent with the measurements.

The L1 and L2-norm errors are listed in the objective function only to display the configuration flexibility. An L1norm error model has an advantage of minimizing the effect of outliers and the ability to create a zero penalty zone around the model value. For the application shown in this paper, the L2-norm was chosen to better distribute the movement of the disturbances. Also, gross error detection reduced the need for improved outlier rejection of the L1-norm.

With a long extended prediction horizon of the controller, it is important to have a reliable future prediction of the disturbance. For this reason, the disturbance value is held constant over the MHE horizon. This single disturbance value is then projected into the future for the control optimization. Allowing the disturbance value to vary from node to node did not produce a reliable future prediction. Additionally, the penalization for changes in the disturbance acts to improve the reliability of the predicted disturbance.

The approach taken in this work is simultaneous solution approach where the objective function and model equations are solved together. The nonlinear DAE model equations are converted to algebraic equations through orthogonal collocation on finite elements. The resulting set of algebraic equations is solved with the NOVA ${ }^{\mathrm{TM}}$ solver.

A drawback to MHE implementation can be summarized as "industrial inertia" due to concerns related to configuration and compute time.

\section{Polymerization Modeling}

The context of the polymerization modeling discussed here is for the purpose of using the model in a nonlinear control application. Additional background is provided in Young [16] and Wilmarth [17]. The gas phase polymerization reactor and plant are modeled with the Polymer Dynamic Modeling System $\left(\mathrm{PDMS}^{\mathrm{TM}}\right)$. The model consists of heat, mass, and species balances with a kinetic mechanism for the polymerization reactor. The kinetic model and method of moments for the polymer properties is based on the work by Ray [18]. In addition to the fluidized bed reactor model, the flowsheet includes a compressor, heat exchanger, vent stream, feed streams, polymer takeoff line for degassing, and dynamic models of the base regulatory control system.

The steady state model, $0=f(\dot{x}=0, x, u, d)$, has 1927 state variables, 764 fixed model inputs, and 1 complementarity condition to account for the variable condensed phase of the heat exchanger. For nonlinear MPC, the model is solved over a time horizon with $\dot{x} \neq 0$. There are a total of 10 MVs and $26 \mathrm{CVs}$, including some pass-through CVs (MVs with CV tuning). The MVs are the feed flows to the reactor, reactor temperature, and vent to the monomer recovery unit. The CVs include polymer properties, reactor pressure, reactor ethylene partial pressure, and process constraints. The following discussion focuses on feedback formulation in relation to improving the prediction of the effect of MV changes on the dynamic and final values for the CVs. A diagram of the flowsheet model is shown in Figure 1.

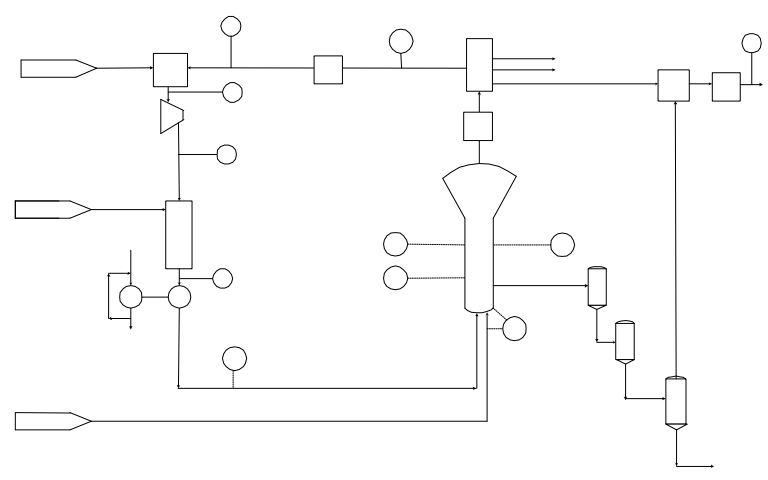

Fig. 1. Flowsheet of the polyethylene plant model.

A complete configuration for feedback via IDF $^{\mathrm{TM}^{\mathrm{i}}}$ is implemented within the application as practically $\mathrm{IDF}^{\mathrm{TM}}$ represents a fall-back layer. There are $27 \mathrm{IDF}^{\mathrm{TM}}$ pairs that couple measured state errors to model disturbance variables. $\mathrm{IDF}^{\mathrm{TM}}$ actively manages 19 disturbances, with backup configuration for the $8 \mathrm{MHE}$ disturbances. Note that the number of $\mathrm{IDF}^{\mathrm{TM}}$ pairs is larger than the number of CVs. This arises as some CVs are duplicated as measured states and matched by adjusting input disturbances, which are tuned less aggressively. Aggressive tuning on output biases is generally incorporated for $\mathrm{CVs}$ explicitly exposed to the controller. Also, note that the input disturbances are abstractly set within the model to minimize the model dynamics with respect to the model measured state. This is done to also allow for more aggressive tuning of $\mathrm{IDF}^{\mathrm{TM}}$.

Due to the choice of MVs, many of the unmeasured input disturbances are abstract flows to and from the cycle gas loop to enable matching of composition and pressure. This includes input disturbance flows for both inert components and reacting components. The inert compositions are nearly integrating variables because they are not consumed in the reaction. Practically, hydrogen composition has some characteristics of these inerts components as it takes significant time to react away given the concentration in the cycle gas. The dynamics for inerts and hydrogen described here are less well-suited for best performance of $\mathrm{IDF}^{\mathrm{TM}}$. In addition, there are some known process phenomena that are not encapsulated within the originally implemented model. These phenomena relate to adsorption and desorption within the polymer bed of heavy hydrocarbon components. Taking these issues into consideration, one could expect that the $\mathrm{IDF}^{\mathrm{TM}}$ feedback on the relevant input disturbances may lead to oscillation. As such, 8 of these input disturbances were defined to be updated by MHE. As such, this simplified version of MHE is structured to allow a direct comparison to $\mathrm{IDF}^{\mathrm{TM}}$.

For the MHE optimization problem, the DAE model is converted to an algebraic model through orthogonal collocation. Figure 2 shows the collocation structure with 6 cubic splines of different time lengths. The derivatives, $\dot{x}$, are approximated by differentiating the splines at the collocation points. At convergence the equation residuals are 
exactly satisfied only at the collocation nodes. The collocated model has a total of 46,870 variables, 18 complementarity conditions, and a Jacobian density for non-zero values of $0.01 \%$.

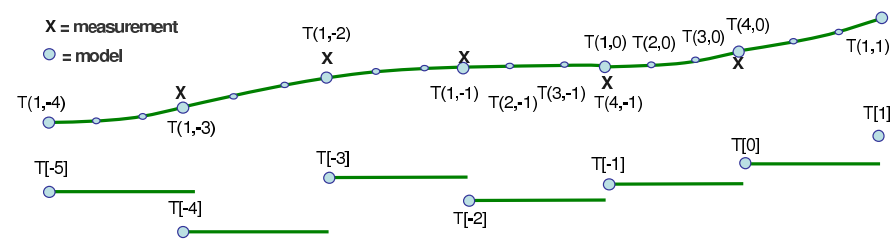

Fig. 2. Orthogonal collocation on finite elements provides an estimate of the state derivatives at the nodes along the time horizon. The state variables are continuous whereas the discrete independent variables are constant over each cubic spline.

As configured, the MHE optimization problem contributes an objective function and 8 degrees of freedom for the 8 disturbance variables referenced in Table I. These disturbances are configured to move only on the first collocation point of the first interval in the horizon. Because MHE does not require one to one pairing of disturbance variables and measured states, additional measurements may be included in the objective function without adding disturbance variables. These measured states are comonomer (comonomer A and/or comonomer B) to monomer (ethylene) mole ratios in the reactor. For control of properties, it is more important to match this ratio rather than the absolute mole fraction of either. This is reflected in the higher weighting given to these mole ratios.

TABLE I

IDF $^{\text {TM }}$ PAIRING AND TUNING

\begin{tabular}{|c|c|c|c|}
\hline Measured State & Disturbance Variable & $K$ & $\begin{array}{c}\tau_{I} \\
\text { (min) }\end{array}$ \\
\hline Ethylene mol\% & Ethylene Feed Bias & 3 & 5 \\
\hline Comonomer A mol\% & Comonomer A Feed Bias & 2 & 5 \\
\hline Comonomer B mol\% & Comonomer B Feed Bias & 4 & 5 \\
\hline $\begin{array}{c}\text { Condensing } \\
\text { Agent mol\% }\end{array}$ & $\begin{array}{c}\text { Condensing Agent } \\
\text { Feed Bias }\end{array}$ & 0.25 & 5 \\
\hline Hydrogen mol\% & Hydrogen Feed Bias & 1 & 5 \\
\hline Pressure & Vent Flow Bias & 50 & 5 \\
\hline Production Rate & Catalyst Activity & 0.0001 & 10 \\
\hline Cooler Temp & Heat Bias & 0.5 & 10 \\
\hline
\end{tabular}

The L1-norm objective function is more tolerant of outliers, but for this application the measured states of interest are filtered with gross error detection. In addition, the problem can be configured such that if a bad measurement is detected, the primary associated disturbance is no longer a degree of freedom. For this MHE problem, there is significant interaction between selected input disturbances and measured states. As a consequence, the L2-norm for the measurement error was chosen as the objective function in order to smear the movement of the input disturbances.

The tuning parameters were set through the following strategy. First, the relative importance of the measured states was defined and weights set such that the reference weight was 1 . All tuning matrices are diagonal in this application.
Weights on the measured states were increased across the board, for selected intervals of the horizon. This weighting reflected the desire to have current states match the currently measured values more closely. Then, typical steady state gains from the model were used to determine initial values for the regularization weights (input weights) on movement of disturbances with the intention of restricting moves compared to no weight. The predictions were monitored each day and the regularization weights adjusted. A half/double rule was used to change the regularization weights and influence the relative movement of the disturbances. After 3 days of online tuning, performance would be judged optimal for the selected horizon. Horizons of 30,50 , and 70 minutes were evaluated and a horizon of 50 minutes was selected. For the 30 minute horizon, there was judged to be unnecessary movement of the disturbances. The tuning parameters or weights on the squared measurement errors and disturbance regularization weights are shown in Table II.

TABLE II

MHE TUNING

\begin{tabular}{|c|c|}
\hline Measured State & Weight \\
\hline Rx Ethylene mol\% & 1.0 \\
\hline Rx Comonomer A mol\% & 0.1 \\
\hline Rx Comonomer B mol\% & 0.1 \\
\hline Rx Condensing Agent mol\% & 1.0 \\
\hline Rx Hydrogen mol\% & 0.1 \\
\hline Rx Pressure & 4.0 \\
\hline Production Rate & 1.0 \\
\hline Cooler Temp & 1.0 \\
\hline Rx ComA/C2 Mole Ratio & 4.0 \\
\hline Rx ComB/C2 Mole Ratio & 4.0 \\
\hline & \\
\hline Input Disturbance & Weight \\
\hline Ethylene Feed Bias & $\frac{1}{200}$ \\
\hline Comonomer A Feed Bias & $\frac{1}{15}$ \\
\hline Condensing Agent Feed Bias & $\frac{1}{7}$ \\
\hline Comonomer B Feed Bias & $\frac{1}{15}$ \\
\hline Hydrogen Feed Bias & $\frac{1}{40}$ \\
\hline Catalyst Activity & $\frac{1}{3}$ \\
\hline Heat Loss Bias & $\frac{1}{50}$ \\
\hline Vent Bias & $\frac{1}{10000}$ \\
\hline
\end{tabular}

The MHE problem typically solves in 2-3 iterations and in under 30 seconds of CPU time on a $3.4 \mathrm{GHz} \mathrm{PC}$ computer. Once converged, the estimate of the current states and disturbance variables are transferred as initial conditions for the control optimization. In the event that MHE does not converge or converges with an infeasible solution, $\mathrm{IDF}^{\mathrm{TM}}$ automatically initiates.

\section{RESULTS}

The results shown in this paper are from testing performed on an industrial gas phase polymerization plant with online process measurements, occasionally corrupt or missing data, and polymer grade and production rate transitions. The process model is run real-time in parallel to the plant, but no optimal control results are transferred to the process (open loop control during model validation prior to controller commissioning). The comparison is between the two modes 
of feedback noted in Section III with one mode exclusive of MHE and the other mode with $\mathrm{IDF}^{\mathrm{TM}}$ and MHE. The $\mathrm{IDF}^{\mathrm{TM}}$ and MHE results are shown for pressure (Figures 3 and 4) and reactor condensing agent mole fraction (Figures 5 and 6). All results are normalized to the average measured values for the $\mathrm{IDF}^{\mathrm{TM}}$ period. Both $\mathrm{IDF}^{\mathrm{TM}}$ and MHE periods are shown for 2 days of plant testing, with different time periods for each. A better comparison would be against the same plant data, but performance indicators can still be compared with these results.
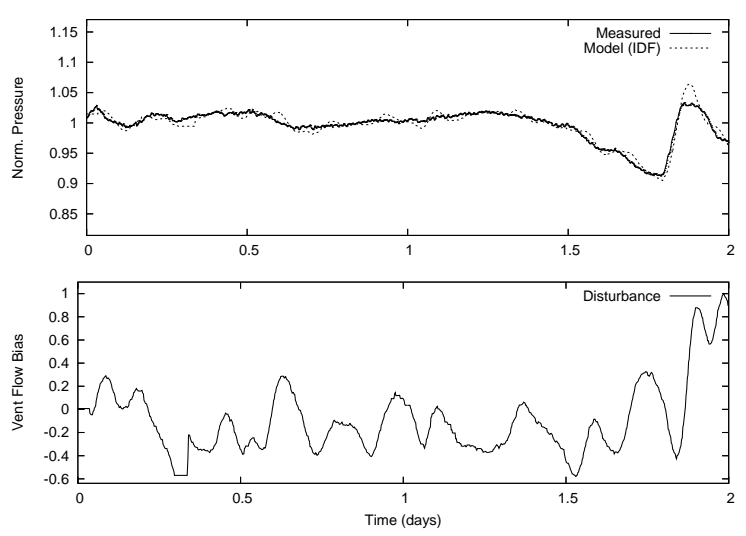

Fig. 3. $\mathrm{IDF}^{\mathrm{TM}}$ for reactor pressure.
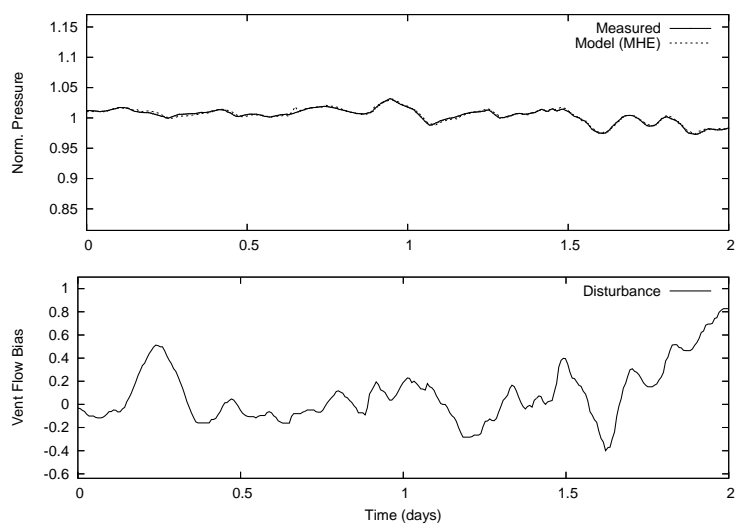

Fig. 4. MHE for reactor pressure.

Two performance indicators for the feedback techniques are:

1) Normalized variance of the model error

2) Variance of the disturbance variable

As a generalization, each indicator is a separate quantitative measure of the feedback effectiveness. Indicator \#1 displays the estimator's ability to track the process (see Table III). Indicator \#2 displays the ability to track the process without aggressive movement of the disturbance variables (see Table IV). The feedback can be tuned aggressively to improve indicator \#1. However, aggressive feedback will increase indicator \#2 and lead to poor future predictions in the subsequent control problem. Indicator \#1 and \#2 are performance measures for state and disturbance tracking, respectively.
TABLE III

Normalized Standard DeViation of Model ERror for the Data SHOWN IN FIGURES 3 TO 6

\begin{tabular}{|c|c|c|c|}
\hline & IDF $^{\text {TM }}$ & MHE & MHE Improvement \\
\hline Production Rate & 0.0275 & 0.0113 & $143 \%$ \\
\hline Pressure & 0.0083 & 0.0019 & $332 \%$ \\
\hline Ethylene mol\% & 0.0059 & 0.0029 & $108 \%$ \\
\hline Hydrogen mol\% & 0.1034 & 0.0026 & $3909 \%$ \\
\hline Condensing Agent mol\% & 0.0489 & 0.0014 & $3288 \%$ \\
\hline
\end{tabular}

TABLE IV

Disturbance Variance for the Data Period in Figures 3 to 6

\begin{tabular}{|c|c|c|c|}
\hline Disturbance Variable & IDF $^{\mathrm{TM}}$ & MHE & MHE Improvement \\
\hline Catalyst Activity & 0.08 & 0.14 & $-43 \%$ \\
\hline Vent Bias & 278.53 & 168.24 & $66 \%$ \\
\hline Ethylene Feed Bias & 5.28 & 4.47 & $18 \%$ \\
\hline Hydrogen Feed Bias & 2.36 & 0.52 & $354 \%$ \\
\hline Condensing Agent Feed Bias & 2.63 & 0.41 & $537 \%$ \\
\hline
\end{tabular}

The performance indicators for MHE are better than for $\mathrm{IDF}^{\mathrm{TM}}$ with the exception of indicator \#2 for catalyst activity. It is speculated that this is due to the production rate increase that occurred during MHE testing, but not during IDF testing. During this time, there was an unmodeled shift in the production rate that was adjusted by catalyst activity.

The improvements evident for the condensing agent and hydrogen are consistent with the discussion in Section III. The condensing agent is an inert that allows increases in production rate by removing the cooling capacity bottleneck. The nearly integrating behavior of the condensing agent mole fraction can be observed in Figure 6. The condensing agent is added to increase the scaled mole fraction from 0.23 to 0.48 . Over the course of the next day, the mole fraction decreases to 0.25 as the condensing agent gradually leaves the system. Although not inert, the hydrogen mole fraction also behaves like an integrating variable because the consumption reaction and losses are low compared to the quantity in the cycle gas. The improved estimation of these two states suggests that MHE is particularly suited for disturbance variables that have integrating effect on the measured state.
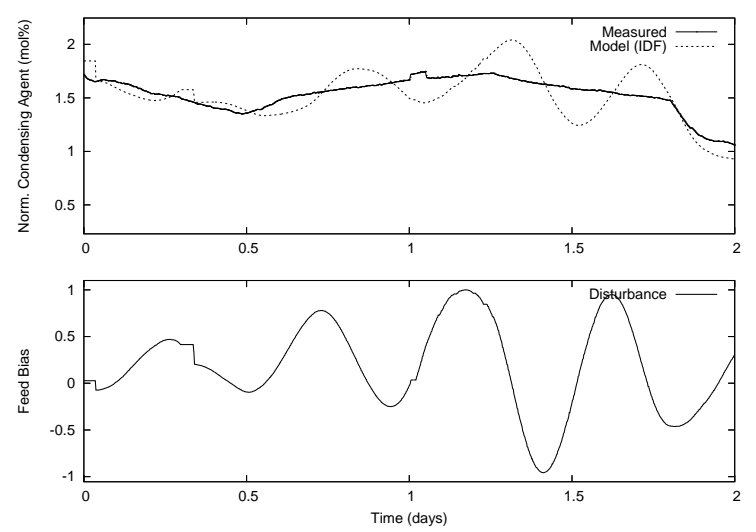

Fig. 5. $\mathrm{IDF}^{\mathrm{TM}}$ for reactor condensing agent mole fraction. 

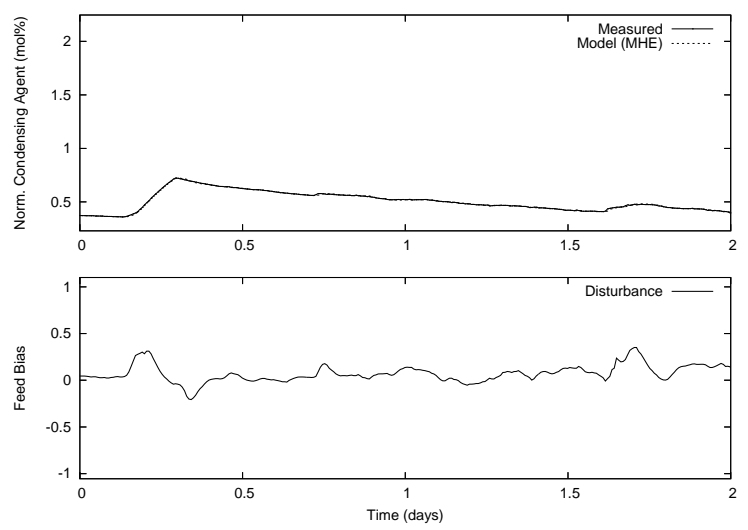

Fig. 6. MHE for reactor condensing agent mole fraction.

\section{Conclusions}

For the selected MVs for this control problem, MHE facilitates improved state estimation compared to $\mathrm{IDF}^{\mathrm{TM}}$ only. This assessment is based on reduced variability of model error and generally reduced movement of disturbance variables. In fact, the project team associated the use of MHE with enabling the use of feed flows as MVs for this control problem. This benefit was gained by improving the model predictions without improving the modeling of the dynamic adsorption and desorption phenomena.

MHE outperforms the current industrial practice of output or integrated bias feedback. This conclusion is based on a comparison with $\mathrm{IDF}^{\mathrm{TM}}$, which is a more capable and generalized form of input and output disturbance adjustment. However, MHE is not always the best choice for feedback just as MPC is not always better than PID control. For situations where the underlying dynamic model is sufficiently accurate, $\mathrm{IDF}^{\mathrm{TM}}$ is comparable to MHE for state estimation. MHE may require additional configuration/setup time and/or the controller scan time with MHE may need to be extended to account for the additional computation time. Where these issues are of relevance, $\mathrm{IDF}^{\mathrm{TM}}$ should be considered.

Besides better tracking of the process with less variability in prediction error, a major driving force for MHE application is the use of less sophisticated models for predictive analysis (in this case, nonlinear MPC). MHE enables a simpler model to be used with an improved selection of MVs and additional CVs for an improved control application. Marquardt stated that the major bottleneck to successful nonlinear MPC is the lack of reliable first-principles or hybrid models [19]. With MHE this bottleneck is reduced by allowing simpler models to be used in more advanced applications.

This paper is one of the few publications focusing on state estimation, particularly MHE, for application of nonlinear MPC in an industrial setting. Future focus will center on more efficient computation of the MHE step and wider use of MHE to facilitate simpler but rigorous chemical engineering models within nonlinear control applications.

\section{ACKNOWLEDGMENTS}

The authors would like to gratefully acknowledge the funding and support from SASOL, South Africa, contributions from Dr. Jeff Renfro, and support from PAS management.

\section{REFERENCES}

[1] M. Soroush, "State and parameter estimations and their applications in process control," Computers and Chemical Engineering, vol. 23, pp. $229-245,1998$

[2] K. R. Muske and T. A. Badgwell, "Disturbance modeling for offsetfree linear model predictive control," Journal of Process Control, vol. 12, pp. 617-632, 2002.

[3] G. Pannocchia, "Robust disturbance modeling for model predictive control with application to multivariable ill-conditioned processes," Journal of Process Control, vol. 13, no. 8, pp. 693-701, 2003.

[4] S. Qin and T. Badgwell, Nonlinear Model Predictive Control. Boston, MA: Birkhäuser Verlag, 2000, ch. An overview of nonlinear model predictive control applications, pp. 369-392.

[5] J. Froisy, "Model predictive control - building a bridge between theory and practice," in Proc. of the CPC VII, Lake Louise, Alberta, Canada, Jan. 2006.

[6] E. Haseltine and J. Rawlings, "Critical evaluation of extended Kalman filtering and moving-horizon estimation," Ind. Eng. Chem. Res., vol. 60, no. 10, pp. 2627-2641, April 2005.

[7] S. Jang, B. Joseph, and H. Mukai, "Comparison of two approaches to on-line parameter and state estimation of nonlinear systems," Ind. Eng. Chem. Process Des. Dev., vol. 25, pp. 809-814, 1986.

[8] M. Liebman, T. Edgar, and L. Lasdon, "Efficient data reconciliation and estimation for dynamic processes using nonlinear programming techniques," Computers and Chemical Engineering, vol. 16, pp. $963-$ 986, 1992.

[9] T. Soderstrom, T. Edgar, L. Russo, and R. Young, "Industrial application of a large-scale dynamic data reconciliation strategy," Industrial and Engineering Chemistry Research, vol. 39, pp. 1683-1693, 2000.

[10] P. Moraal and J. Grizzle, "Observer design for nonlinear systems with discrete-time measurements," IEEE Transactions on Automatic Control, vol. 40, no. 3, pp. 395-404, 1995.

[11] C. Rao, J. Rawlings, and J. Lee, "Constrained linear state estimation - a moving horizon approach," Automatica, vol. 37, pp. 1619-1628, 2001.

[12] J. Albuquerque and L. Biegler, "Decomposition algorithms for online estimation with nonlinear models," Computers and Chemical Engineering, vol. 19, no. 10, pp. 1031-1039, 1995.

[13] J. Hedengren and T. Edgar, "Moving horizon estimation - the explicit solution," in Proc. of the CPC VII, Lake Louise, Alberta, Canada, Jan. 2006.

[14] L. Russo and R. Young, "Moving-horizon state estimation applied to an industrial polymerization process," in In Proc. American Control Conf., San Diego, CA, 1999, pp. 1129-1133.

[15] T. Binder, L. Blank, H. Bock, R. Burlisch, W. Dahmen, M. Diehl, T. Kronseder, W. Marquardt, J. Schlöder, and O. Stryk, Online Optimization of Large Scale Systems. Springer-Verlag Berlin Heidelberg, 2001 , ch. Introduction to model based optimization of chemical processes on moving horizons, pp. 295-339.

[16] R. Young, "Evolution of an industrial nonlinear model controller," in Proc. of the CPC VI, Tucson, AZ, Jan. 2001.

[17] T. Wilmarth, R. Wuyts, J. Crosby, R. Wagler, J. Renfro, and J. Goodman, "Implementing non-linear model predictive control," Petrochemicals and Gas Processing, Spring 2002.

[18] W. Ray, "On the mathematical modeling of polymerization reactors," J. Macromol. Sci.-Revs. Macromol. Chem., vol. C8(1), pp. 1-56, 1972.

[19] W. Marquardt, "Nonlinear model reduction for optimization based control of transient chemical processes," in Proc. CPC VI, ser. AIChE Symposium Series, vol. 98, no. 326, 2001, pp. 30-60. 